\begin{tabular}{|c|c|}
\hline Title & $\begin{array}{l}\text { Detection of } \mathrm{OH} \text { radicals in atmospheric-pressure plasmajet by evanescent-wave laser-induced fluorescence } \\
\text { spectroscopy }\end{array}$ \\
\hline Author(s) & Sasaki, K.; Hishida, Y.; Sadeghi, N. \\
\hline Citation & $\begin{array}{l}\text { Journal of Instrumentation, 14(11), C11016 } \\
\text { https://doi.org/10.1088/1748 0221/14/11/C11016 }\end{array}$ \\
\hline Issue Date & $2019-11$ \\
\hline Doc URL & http:/hdl.handle.net/2115/79643 \\
\hline Rights & $\begin{array}{l}\text { This A ccepted Manuscript is available for reuse under a CC BY-NC-ND } 3.0 \text { licence after the } 12 \text { month embargo period } \\
\text { provided that all the terms of the licence are adhered to }\end{array}$ \\
\hline Rights(URL) & https://creativecommons.org/icenses/by-nc-nd/3.0/ \\
\hline Type & article (author version) \\
\hline File Information & EvanescentLIF_final.pdf \\
\hline
\end{tabular}

Instructions for use 


\title{
Detection of $\mathrm{OH}$ radicals in atmospheric-pressure plasma jet by evanescent-wave laser-induced fluorescence spectroscopy
}

\author{
Koichi Sasaki, ${ }^{a, 1}$ Yuto Hishida, ${ }^{a}$ Nader Sadeghi ${ }^{b}$ \\ ${ }^{a}$ Division of Quantum Science and Engineering, Hokkaido University, Kita 13, Nishi 8, Kita-ku, Sapporo \\ 060-8628, Japan \\ ${ }^{b}$ LIPhy (UMR5588) and LTM (UMR5129), Université de Grenoble-Alpes and CNRS, Grenoble, France \\ E-mail: sasaki@qe.eng.hokudai.ac.jp
}

\begin{abstract}
This paper reports the signal and noise in the detection of $\mathrm{OH}$ radicals by evanescentwave laser-induced fluorescence (EW-LIF) spectroscopy. We adopted this method to detect $\mathrm{OH}$ radicals, which were produced within an atmospheric-pressure argon plasma jet, in the vicinity of a quartz surface. The dominant noise was the stray laser light which could not be eliminated by using a monochromator and an interference filter. This was because the detection optics looked at the total reflection point of the laser beam in the EW-LIF spectroscopy. It was impossible to detect the LIF signal in the duration of the laser pulse because of the intense stray light. On the other hand, thanks to the slow decay of the LIF intensity, we succeeded in detecting the LIF signal after the laser pulse. The intensity ratio between the LIF signal originated from the bulk plasma and the one induced by EW near the surface was 5000-10000, which can be explained by the difference in the observation volumes. The proportionality between the LIF intensities from the bulk plasma and the surface vicinity, when changing the discharge conditions, suggests that the surface loss probability of $\mathrm{OH}$ on the quartz surface was not affected by these changes.
\end{abstract}

KeYwORDs: Plasma diagnostics - interferometry, spectroscopy and imaging

\footnotetext{
${ }^{1}$ Corresponding author.
} 


\section{Contents}

1 Introduction $\quad 1$

2 Experiment 2

3 Results $\quad 3$

4 Discussion $\quad 5$

5 Conclusions $\quad 7$

\section{Introduction}

Atmospheric-pressure plasmas are studied widely with the intention of applying them to medicine, agriculture, and environmental technologies. Reactive radicals interact with surfaces of solids or liquids in many applications, and in this case, an important value is the radical density in the vicinity of the surfaces. In general, the radical density decreases toward the surface if the surface loss probability is not zero, and the radical density in the vicinity of the surface is significantly affected by the surface loss probability $[1,2]$. The spatial range with the decreasing radical density is determined by the mean free path [3,4]. In conventional low-pressure plasmas, it is meaningful to measure the radical density at a macroscopic distance (for example a few millimeters) from the surface because of the long mean free path. However, in atmospheric pressure plasmas, the density decrease in the surface vicinity occurs within typically $10 \mu \mathrm{m}$, a too short distance for carrying out space-resolved density measurement experiments.

In this work, we examined the usefulness of evanescent-wave laser induced fluorescence (EWLIF) spectroscopy for the measurement of $\mathrm{OH}$ radical density in the vicinity of a quartz surface. LIF spectroscopy is widely used for the measurement of radical densities in reactive plasmas. The observation volume or the spatial resolution in conventional LIF is determined by the cross region between the tunable laser beam and the image beam of the detection optics, and it is typically 1 $\mathrm{mm}^{3}$. Hence, it is impossible to detect LIF from only the region in the vicinity $(\leq 10 \mu \mathrm{m})$ of the surface. The evanescent wave (EW) [5] is an oscillating electromagnetic field that does not propagate into the free space. The energy of EW is spatially concentrated in the vicinity of the boundary between two mediums with different refractive indexes. EW is generated when a laser beam is injected into the boundary at an incident angle which is greater than the critical angle. In the present experimental condition, the penetration depth of EW is roughly $140 \mathrm{~nm}$ [5]. Therefore, if we can detect LIF induced by the EW laser field, the LIF intensity can provide the radical density in the vicinity of the boundary.

Bordo and coworkers investigated the fluorescence spectrum of $\mathrm{Na}$ atoms excited by an evanescent wave at a glass prism surface [6]. The EW-LIF spectroscopy was adopted to plasma diagnostics 


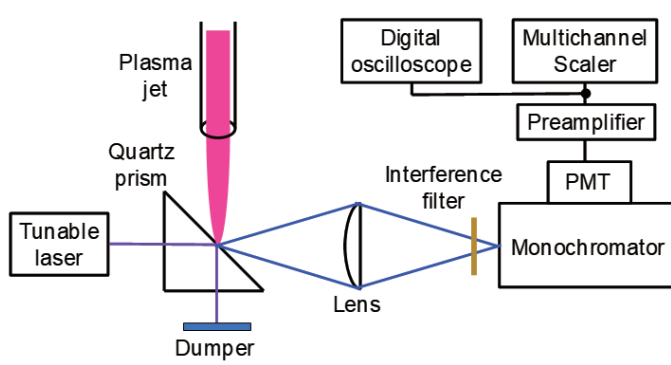

(a)

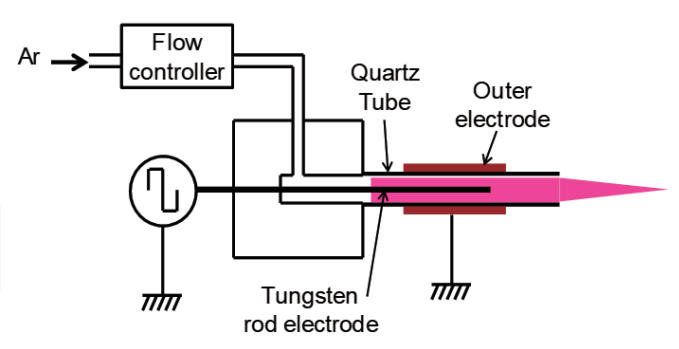

(b)

Figure 1. Schematic of the experimental setup. (a) Optical configuration of evanescent-wave laser induced fluorescence spectroscopy. (b) Details of the atmospheric-pressure argon plasma jet.

by Sakurai and coworkers [7, 8]. They measured the density of argon metastable atoms in the vicinity of a dielectric surface in a dielectric barrier discharge. However, to our best knowledge, the EW-LIF spectroscopy has not been used for measuring the radical density in the vicinity of a surface. In this work, we tried the EW-LIF spectroscopy of $\mathrm{OH}$ radicals in the vicinity of a quartz surface. We analyzed the EW-LIF signal intensity and the origin of the noise associated to it, in the detection of $\mathrm{OH}$ radicals produced using an atmospheric-pressure argon plasma jet.

\section{Experiment}

We employed two excitation schemes for the LIF detection of $\mathrm{OH}$ radicals. One was the excitation from the $\mathrm{X}^{2} \Pi\left(v^{\prime \prime}=0\right)$ to $\mathrm{A}^{2} \Sigma^{+}\left(v^{\prime}=1\right)$ states at a wavelength of $283.03 \mathrm{~nm}$. An optical parametric oscillator (OPO laser) was used for this excitation. The energy of the OPO laser pulse was approximately $1 \mathrm{~mJ}$. The other scheme was the excitation from the $\mathrm{X}^{2} \Pi\left(v^{\prime \prime}=0\right)$ to $\mathrm{A}^{2} \Sigma^{+}\left(v^{\prime}=2\right)$ states at a wavelength of $261.68 \mathrm{~nm}$. A dye laser was used for this excitation. The energy of the dye laser pulse was approximately $5 \mathrm{~mJ}$. In both the excitation schemes, we detected the fluorescence by the transition from $\mathrm{A}^{2} \Sigma^{+}\left(v^{\prime}=0\right)$ to $\mathrm{X}^{2} \Pi\left(v^{\prime \prime}=0\right)$ at around $309 \mathrm{~nm}$. The $\mathrm{A}^{2} \Sigma^{+}\left(v^{\prime}=0\right)$ state was populated by the vibrational energy transfer from the $\mathrm{A}^{2} \Sigma^{+}\left(v^{\prime}=1\right)$ and $\mathrm{A}^{2} \Sigma^{+}\left(v^{\prime}=2\right)$ states.

The experimental setup is shown in Fig. 1(a). The unfocused tunable laser beam was injected into a quartz prism, and the laser beam experienced a total reflection on the quartz-to-air interface. The polarizations of the laser beam were $p$ and $s$ in the excitations to the $\mathrm{A}^{2} \Sigma^{+}\left(v^{\prime}=1\right)$ and $\mathrm{A}^{2} \Sigma^{+}\left(v^{\prime}=2\right)$ states, respectively. The detection system was composed of a monochromator and a photomultiplier tube (PMT). The fluorescence from the total reflection point was projected onto the entrance slit of the monochromator using a quartz lens. The monochromator had a focal length of $30 \mathrm{~cm}$ and a diffraction grating with 1200 grooves $/ \mathrm{mm}$. The inverse dispersion was 3 $\mathrm{nm} / \mathrm{mm}$. An interference filter, centered at $310 \mathrm{~nm}$ with $\pm 5 \mathrm{~nm}$ bandpass, was placed in front of the monochromator to block the stray laser light. The attenuation factor of the interference filter was approximately $1 / 10$ at the laser wavelength. The electrical signal from PMT was amplified by factor 20 using a preamplifier with an input resistor of $50 \Omega$. The amplified signal was connected to a digital oscilloscope (DO) and a multichannel scaler (MS; Stanford Research SR430) with a binning width of $40 \mathrm{~ns}$. We realized the temporally resolved photon counting detection of the 

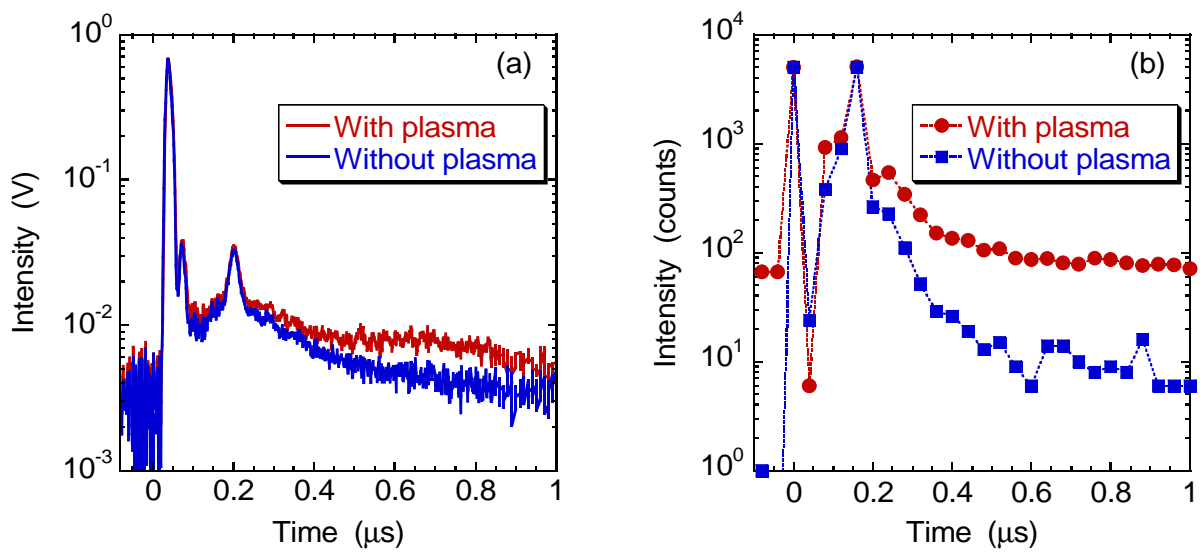

Figure 2. Signals observed in the presence and absence of the plasma jet. (a) was observed using the digital oscilloscope after averaging 128 laser pulses. (b) was observed using the multichannel scaler by counting photons for 5000 laser pulses. The $\mathrm{A}^{2} \Sigma^{+}\left(v^{\prime}=2\right)$ state was excited using the dye laser.

fluorescence using MS. The small resistor of $50 \Omega$ was necessary to realize fast photon pulses which were suitable for the counting detection. We adjusted the discrimination level carefully to achieve a high signal-to-noise ratio.

The total reflection point was exposed to an argon plasma jet shown in Fig. 1(b). In this plasma jet, argon was flowed along a quartz tube of $4 \mathrm{~mm}$ inner diameter. A tungsten rod electrode was placed at the center of the quartz tube, and it was connected to a bipolar high-voltage power supply with a rectangular waveform. The amplitude and the frequency of the high voltage were $2-7 \mathrm{kV}$ and $2-4 \mathrm{kHz}$, respectively. The ground electrode was wrapped on the outer surface of the quartz tube. A dielectric barrier discharge was obtained inside the quartz tube, and a plasma jet with a length of approximately $20 \mathrm{~mm}$ was ejected from the end of the quartz tube. The distance between the end of the quartz tube and the prism surface was approximately $15 \mathrm{~mm}$.

\section{Results}

The signals observed in the presence and absence of the plasma jet are shown in Fig. 2. The waveforms shown in Fig. 2(a) were observed using DO after averaging 128 pulses. The waveform shown in Fig. 2(b) was observed using MS by counting photons for 5000 laser pulses. The discrimination level in the photon counting observation was set at $15 \mathrm{mV}$. The voltage and the frequency of the power supply for producing the plasma jet were $5 \mathrm{kV}$ and $4 \mathrm{kHz}$, respectively. The bias voltage of PMT was $-700 \mathrm{~V}$, and the slit width of the monochromator was $1 \mathrm{~mm}$. The dye laser was used for exciting $\mathrm{OH}$ radicals to the $\mathrm{A}^{2} \Sigma^{+}\left(v^{\prime}=2\right)$ state.

The signal observed in the absence of the plasma jet was dominated by the stray laser light at $t \simeq 0 \mu \mathrm{s}$. Although the stray laser light was attenuated by the interference filter and the monochromator, it was so intense that a spiky pulse was observed during each laser pulse. Thus the light intensity recorded using MS was 5000 counts (the pulse was detected in every laser shot), as shown in Fig. 2(b). In addition to the stray laser light at $t \simeq 0 \mu \mathrm{s}$, we observed a second peak at $t \simeq 0.16 \mu \mathrm{s}$ in the absence of the plasma jet. We observed the same signal when we detuned 


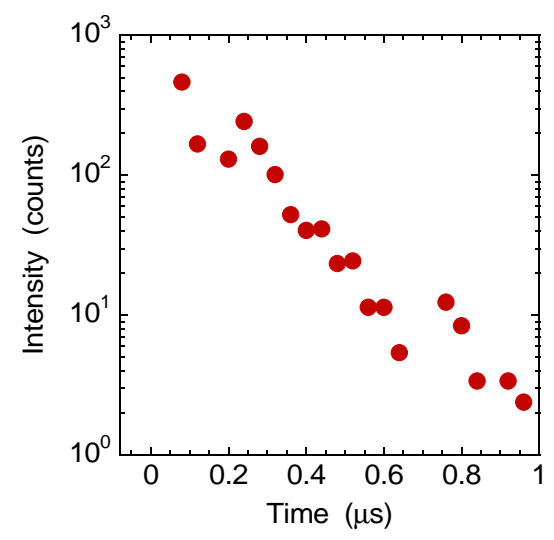

Figure 3. Temporal variation of the EW-LIF intensity, which was deduced from Fig. 2(b) by subtracting the components of stray laser light and the self-emission of the plasma jet.

the laser wavelength from the resonant excitation wavelength of $\mathrm{OH}$ in the presence of the plasma jet. When recorded using DO, the amplitude of the 2nd pulsed noise was approximately $5 \%$ of the stray laser light at $t=0 \mu$ s (Fig. 2(a)). But it was detected in every laser shot since its amplitude exceeded the discrimination level (Fig. 2(b)). No signals were detected when we examined the fluorescence spectrum at $t \simeq 0.16 \mu \mathrm{s}$ in the absence of the plasma using a spectrograph combined with a charge-coupled device camera with a gated image intensifier, indicating that the second pulsed noise was not optical emission. Therefore, we speculated that the second pulsed noise was due to the electrical response of PMT after the delivery of the intense pulsed current of $\sim 1 \mathrm{~mA}$ at $t \simeq 0 \mu \mathrm{s}$. The slow tail component observed after the second pulsed noise may also be due to the electrical response of PMT.

An enhancement of the signal intensity was observed when the plasma jet was switched on. This extra-signal comes, partly, from the self-emission of the plasma jet, but also from EW-LIF. The increase was much smaller in the signal recorded with DO than that recorded with MS. The level of the self-emission is seen in Fig. 2(b) at $t \leq 0 \mu \mathrm{s}$. It was impossible to deduce the EW-LIF component from Fig. 2(a) because of the insufficient signal-to-noise ratio. On the other hand, we deduced the EW-LIF component from Fig. 2(b), as shown in Fig. 3, by subtracting the stray laser light and the self-emission of the plasma jet. It is noted that the signal at $t \leq 0.16 \mu$ s is not reliable. The data points of the EW-LIF intensity are quite noisy, but an exponential decay with a time constant of $0.17 \mu$ s can easily be plotted after the second pulsed noise, as shown in Fig. 3.

Figure 4 shows the LIF signal from the volume of the argon plasma jet. In this case, the dye laser beam crossed the plasma jet at a distance of $15 \mathrm{~mm}$ from the end of the quartz tube, and the fluorescence was projected onto the entrance slit of the monochromator. The voltage and the frequency of the power supply for producing the plasma jet were $5 \mathrm{kV}$ and $4 \mathrm{kHz}$, respectively, and the bias voltage of PMT was $-700 \mathrm{~V}$. The same excitation scheme as that of EW-LIF was used in this conventional LIF experiment. When the slit width was $1 \mathrm{~mm}$, a clear LIF signal was observed using DO, as shown by the red curve in Fig. 4(a). In this case, it was impossible to record the LIF intensity using MS, since we observed the overlapping of many photons within the binning width of $40 \mathrm{~ns}$. When we narrowed the slit width to $0.05 \mathrm{~mm}$, as shown by the blue curve in Fig. 4(a), the 

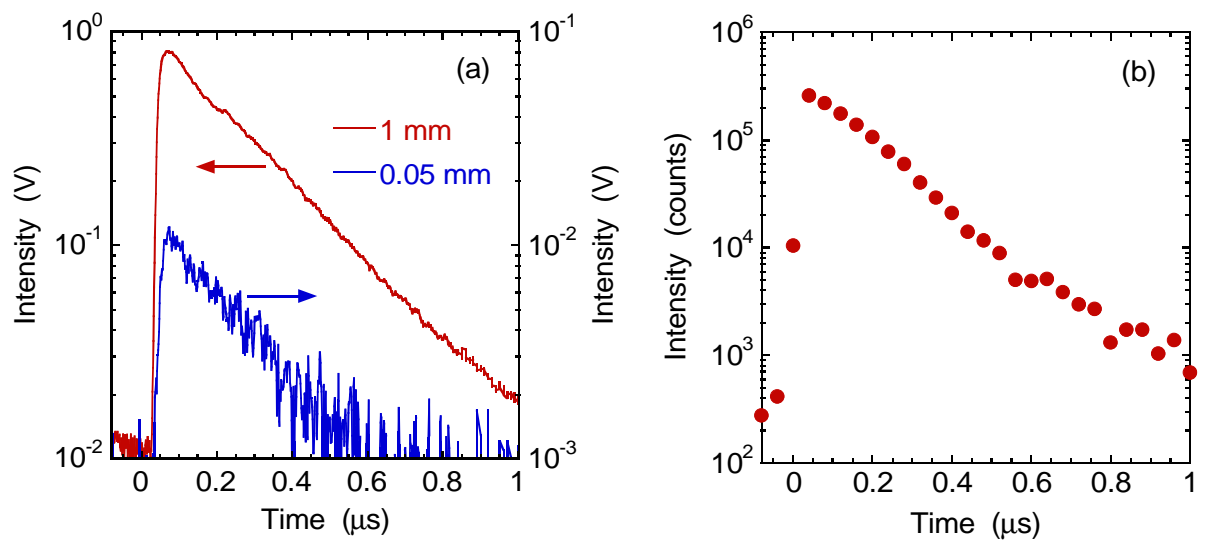

Figure 4. Signals of conventional LIF spectroscopy in the same plasma jet. (a) LIF intensities recorded using the digital oscilloscope. The slit widths of the monochromator were 1 and $0.05 \mathrm{~mm}$. (b) LIF intensity recorded using MS. The slit width of the monochromator was $0.05 \mathrm{~mm}$.

amplitude of the signal recorded using DO was reduced to $1.5 \%$ of the signal observed at the slit width of $1 \mathrm{~mm}$, and in this case it was possible to record the LIF intensity using MS, as shown in Fig. 4(b). The waveform shown in Fig. 4(b) was obtained by counting photons for 5000 laser pulses, and the raw signal was multiplied by a factor of $1 / 0.015$ to reproduce the intensity that should be observed at the slit width of $1 \mathrm{~mm}$. The decay time constant of the exponential decrease shown in Fig. 4(b) is almost the same as that shown in Fig. 3.

The comparison between the fluorescence intensities observed in EW and conventional LIF is shown in Fig. 5. The $\mathrm{OH}$ radical density was varied by changing the discharge voltage, the gas flow rate, and the distance between the end of the quartz tube and the observation position. We used the OPO laser in this experiment, and the scheme of the excitation was from $\mathrm{X}^{2} \Pi\left(v^{\prime \prime}=0\right)$ to $\mathrm{A}^{2} \Sigma^{+}\left(v^{\prime}=1\right)$. As shown in the figure, we observed a proportional relationship between the intensities of EW and conventional LIF.

\section{Discussion}

The most serious difficulty in the EW-LIF spectroscopy was the huge stray light. It was caused by the fact that the detection optics looked at the total reflection point on the quartz prism, as shown in Fig. 1(a). With a perfectly polished prism surface, no stay light should be observed since EW does not propagate into the free space. However, in practice, the scattering of EW is unavoidable due to the non-zero roughness of the prism surface, resulting in a strong stray light from the total reflection point. The difficulty in the optical configuration shown in Fig. 1(a) is that both the signal (LIF) and the noise (the stray laser light) come from the same position, and the detection optics should look at the total reflection point. To observe the EW-LIF signal during the laser pulses, a spectroscopic method that is able to efficiently eliminate the stray laser light is needed. The use of a triple-grating spectrograph [9] could be a solution. In addition, an improved PMT circuit is necessary to eliminate the second pulsed noise $(t \simeq 0.16 \mu \mathrm{s})$ and the tail noise component shown in Fig. 2. 


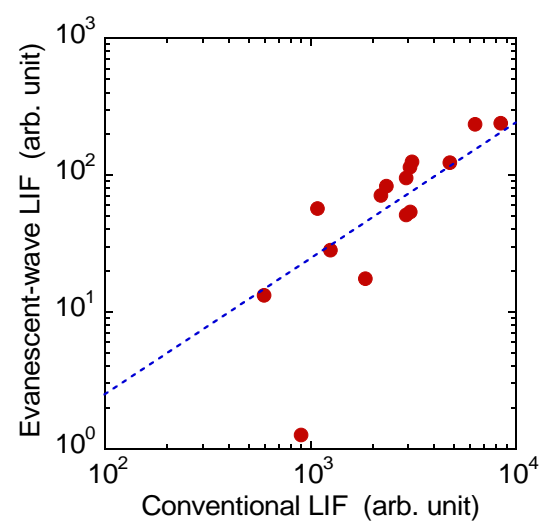

Figure 5. Comparison between the fluorescence intensities observed in EW and conventional LIF. The $\mathrm{A}^{2} \Sigma^{+}\left(v^{\prime}=1\right)$ state was excited using the OPO laser.

The detection of $\mathrm{OH}$ radicals is an adaptable case to the EW-LIF spectroscopy, since the $\mathrm{A}^{2} \Sigma^{+}\left(v^{\prime}=0\right)$ state has a long lifetime. The lifetime of the $\mathrm{A}^{2} \Sigma^{+}\left(v^{\prime}=0\right)$ state is determined by collisional quenching in the atmospheric pressure. According to the rate coefficient of collisional quenching reported by Martini and coworkers [10], the lifetime of $0.17 \mu \mathrm{s}$, which was observed as the decay time constant of the LIF intensities, corresponds to the mixing ratio of $0.4 \%$ from ambient air into the argon flow. The measurement becomes more difficult than the present experiment if the mixing ratio of ambient air is higher.

By comparing Figs. 3 and 4(b), it is understood that the intensity of EW-LIF was weaker than that of conventional LIF by a factor of 400-500. To obtain Fig. 4(b), the LIF signal was attenuated by reducing the slit width of the monochromator to $0.05 \mathrm{~mm}$ and the raw recorded signal was multiplied by a factor $1 / 0.015$. The narrow slit width decreased the observation volume of LIF, resulting in the attenuated LIF intensity. However, it is noted that the narrow slit width also results in a finer spectral width. The spectrum of the $\mathrm{A}^{2} \Sigma^{+}\left(v^{\prime}=0\right)-\mathrm{X}^{2} \Pi\left(v^{\prime \prime}=0\right)$ transition of $\mathrm{OH}$ extends over $\sim 3 \mathrm{~nm}$ if we assume $500 \mathrm{~K}$ for the rotational temperature. The wavelength resolution at a slit width of $1 \mathrm{~mm}$ was $3 \mathrm{~nm}$, while it was $0.15 \mathrm{~nm}$ when the slit width was $0.05 \mathrm{~mm}$. Hence, the conventional LIF intensity would be one order of magnitude stronger if the LIF intensity was reduced using an attenuator but keeping the slit width at $1 \mathrm{~mm}$. Considering the difference in the wavelength resolution, it is speculated that the ratio between the EW and conventional LIF intensities is 5000-10000. This intensity ratio is reasonable. This is because the observation volume in conventional LIF was roughly $1 \mathrm{~mm}^{3}$ (both the spot size of the laser beam and the slit width were $1 \mathrm{~mm}$ ), while in EW-LIF the observation volume was roughly $1 \mathrm{~mm} \times 1 \mathrm{~mm} \times 100 \mathrm{~nm}$. Note that in the $p$-polarized total reflection, the intensity of the EW laser field is equal to that of the incident laser beam [5].

The proportional relationship shown in Fig. 5 was observed between the EW and conventional LIF intensities. This means that the $\mathrm{OH}$ density in the vicinity $(\leq 140 \mathrm{~nm})$ of the quartz surface was proportional to the $\mathrm{OH}$ density in the bulk region of the plasma jet. Since the radical density in the vicinity of the quartz surface is affected by the loss probability on it, the proportional relationship suggests that the surface loss probability was not affected by the discharge conditions in the present 
experiment. In addition, the surface loss probability may be much smaller than unity, since the ratio between the EW and conventional LIF intensities can be explained by the difference in the observation volumes. However, we believe that this is not the universal nature of $\mathrm{OH}$ radicals. We will continue the experiment at different conditions to find more general relationship between the $\mathrm{OH}$ densities in the vicinity of a surface and in the bulk region of the plasma.

\section{Conclusions}

In this work, we examined the usefulness of the EW-LIF spectroscopy for the measurement of $\mathrm{OH}$ radical density in the vicinity of a quartz surface. We succeeded in detecting the EW-LIF signal at $t \geq 0.16 \mu \mathrm{s}$ after the laser pulse. On the other hand, the detection of the EW-LIF signal was impossible in the duration of the tunable laser pulse due to the intense stray laser light. The ratio between the $\mathrm{EW}$ and conventional LIF intensities was reasonable by considering the difference in the observation volumes. A proportional relationship was observed between the EW and conventional LIF intensities, suggesting that the loss probability of $\mathrm{OH}$ radicals on the quartz surface was not affected by the discharge conditions. The conclusion of the present trial is that a method to suppress the stray laser light efficiently is the key technique to apply the EW-LIF spectroscopy to various radical species.

\section{References}

[1] A. D. Tserepi, J. Derouard, J. P. Booth, and N. Sadeghi, $C F_{2}$ kinetics and related mechanisms in the presence of polymers in fluorocarbon plasmas, J. Appl. Phys. 81 (1997) 2124.

[2] N. Nafarizal and K. Sasaki, Sticking probability of Ti atoms in magnetron sputtering deposition evaluated from the spatial distribution of Ti atoms density, J. Vac. Sci. Technol. A 25 (2007) 308.

[3] P. J. Chantry, A simple formula for diffusion calculations involving wall reflection and low density, J. Appl. Phys. 62 (1987) 1141.

[4] P. J. Chantry, A. V. Phelps, and G. J. Schulz, Theory of electron collision experiments at intermediate and high gas densities, Phys. Rev. 152 (1966) 81.

[5] M. Bertolotti, C. Sibilia, and A. M. Guzmán, Evanescent Waves in Optics, Springer (2017).

[6] V. G. Bordo, C. Henkel, A. Lindinger, and H.-G. Rubahn, Evanescent wave fluorescence spectra of Na atoms, Opt. Comm. 137 (1997) 249.

[7] T. Sakurai, Laser-based plasma particle analysis of the surface in a discharge, Plasma Sources Sci. Technol. 16 (2007) S101.

[8] T. Sakurai, T. Kubota, Y. Takahara, Y. Inoue, and H. Hori, Density of Ar metastable atoms on the discharge tube wall measured by evanescent laser spectroscopy, Jpn. J. Appl. Phys. 38 (1999) L590.

[9] A. Kono and K. Nakatani, Efficient multichannel Thomson scattering measurement system for diagnostics of low-temperature plasmas, Rev. Sci. Instrum. 71 (2000) 2716.

[10] L. M. Martini, N. Gatti, G. Dilecce, M. Scotoni, and P Tosi, Rate constants of quenching and vibrational relaxation in the $\mathrm{OH}\left(A^{2} \Sigma^{+}(v=0,1)\right.$, manifold with various colliders, J. Phys. D: Appl. Phys 50 (2017) 114003. 